
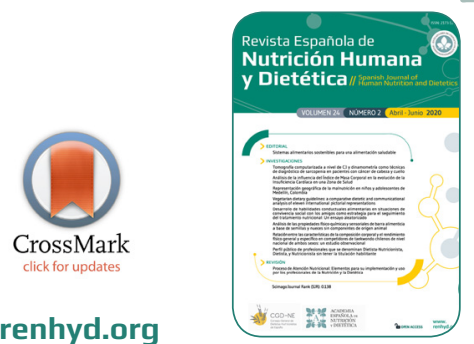

www.renhyd.org

\author{
ARTÍCULO DE INVESTIGACIÓN
}

\title{
Análisis de las propiedades físico-químicas y sensoriales de barra alimenticia a base de semillas y nueces sin componentes de origen animal
}

\author{
Lydia Toscano-Palomar ${ }^{\mathrm{a}, *}$, Gilberto García-Gómez ${ }^{\mathrm{b}}$, Francisco J Gómez-Puentes ${ }^{\mathrm{a}}$, Guillermo \\ Beltrán-González ${ }^{c}$, Iracema G Valenzuela-Espinoza ${ }^{a}$, Jesus M Armenta-Gálvez ${ }^{a}$

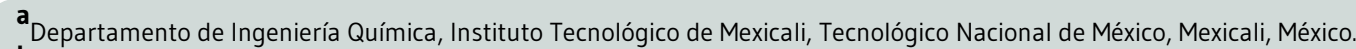 \\ b División de Estudios de Posgrado, Instituto Tecnológico de Mexicali, Tecnológico Nacional de México, Mexicali, México. \\ Instituto de Innovación para la Salud Integral, A. C., Mexicali, México. \\ *toscano.lydia@tmexicali.edu.mx
}

Editor Asignado: Miguel Ángel Lurueña. Comité Editorial de la Revista Española de Nutrición Humana y Dietética. Pamplona, España.

Recibido el 19 de noviembre de 2019; aceptado el 10 de marzo de 2020; publicado el 14 de abril de 2020.

\section{PALABRAS CLAVE}

Alimentos;

Nueces;

Semillas;

Análisis de los

Alimentos;

Valor Nutritivo;

Calidad de los

Alimentos;

Comportamiento

del Consumidor;

Emociones:

Almacenamiento de Alimentos;

Vida útil.
Análisis de las propiedades físico-químicas y sensoriales de barra alimenticia a base de semillas y nueces sin componentes de origen animal

\section{RESUMEN}

Introducción: La necesidad de alimentos nutritivos se ha incrementado por la demanda de un público exigente. Los consumidores, en la actualidad, desean aperitivos que además de satisfacer su apetito, aporten también nutrientes esenciales. Este trabajo tuvo como objetivo la caracterización físico-química de una barra alimenticia elaborada a base de semillas (girasol, ajonjolí, chía y linaza) y nueces (almendra, nuez de la India, corazón de nuez de pecana, pistache y nuez de Castilla entera) libre de componentes de origen animal, como leche de vaca en polvo, ovoalbúmina y/o grasas animales utilizados en barras alimenticias comerciales.

Material y Métodos: Los métodos de análisis próximo, valor energético, vida útil de la barra alimenticia y análisis sensorial y las pruebas de aceptación, se realizaron de acuerdo a métodos estándares de análisis de la Asociación de Químicos Analíticos Oficiales (AOAC por sus siglas en inglés). Todos los análisis se realizaron por triplicado a menos que se indicara de otra manera. Los resultados obtenidos fueron estadísticamente analizados con un $5 \%$ de nivel de significancia para cada prueba.

Resultados: Se analizó su composición próxima ( $27 \%$ proteína, $7,5 \%$ fibra cruda y $4,4 \%$ de azúcares). Estos valores posicionan a este producto dentro de las barras de alto contenido proteico con contenido en azúcar muy bajo si se compara con barras comerciales (20-40\%). Presentó el $45 \%$ de lípidos con el $19 \%$ de aceites saturados y $81 \%$ de insaturados, todos estos derivados de las nueces y semillas. Se cuantificaron minerales esenciales como: calcio, magnesio, potasio, sodio, cobre, hierro, manganeso y zinc. La velocidad de rancidez fue relativamente baja hasta alcanzar el valor máximo permisible de $10 \mathrm{meq} / \mathrm{kg}$ en peróxidos como valor de rancidez a los 9 días de almacenamiento a $40^{\circ} \mathrm{C}$.

Conclusiones: Las barras alimenticias a base de semillas y nueces tuvieron una alta aceptación en pruebas sensoriales de preferencia por público adulto. De acuerdo con estos resultados se presenta un alimento de alto contenido proteico, nutricionalmente completo y estable al almacenamiento. 


\section{KEYWORDS}

Food;

Nuts;

Seeds;

Food Analysis;

Nutritive Value;

Food Quality;

Consumer Behavior;

Emotions;

Food Storage;

Shelf-life.
Analysis of the physical-chemical and sensorial properties of nutritional bars based on seeds and nuts without components of animal origin

\section{ABSTRACT}

Introduction: The need for nutritious food has increased due to the requirement of a demanding public. Consumers today want appetizers that, in addition to satisfying their appetite, also provide essential nutrients. The objective of this work was the physical-chemical characterization of a food bar based on seeds from sunflower, sesame, chia and linseed, and nuts such as almond, cashew, pecan nut kernel, pistachio and whole cashew nut, and free of components of animal origin usually used in commercial food bars (cow's milk powder, ovalbumin and/or animal fats).

Material and Methods: The methods for the proximate composition, the energy value, the shelf life and sensory evaluation and acceptance tests were performed according to the Association Official of Agricultural Chemists (AOAC) standard methods of analysis. All analyzes were performed by triplicate unless otherwise indicated. The results obtained were statistically analyzed with a $5 \%$ level of significance for each test.

Results: Its proximal composition was analyzed (27\% protein, $7.5 \%$ crude fiber and $4.4 \%$ sugars). These values position this product among nutritious bars of high protein content with very low sugar content when compared with commercial bars (20-40\%). He presented $45 \%$ of lipids with $19 \%$ of saturated oils and $81 \%$ of unsaturated oils, all derived from nuts and seeds. Essential minerals were quantified as: calcium, magnesium, potassium, sodium, copper, iron, manganese and zinc. The rancidity rate was relatively low until reaching the maximum value allowed (10meq/kg as total peroxides) at 9 days of storage at $40^{\circ} \mathrm{C}$.

Conclusions: The food bars based on seeds and nuts had a high acceptance by sensory tests of preference for adult public. According to these results, a high-protein, nutritionally complete and storage-stable food is presented.

\section{CITA}

Toscano-Palomar L, García-Gómez G, Gómez-Puentes FJ, Beltrán-González G, Valenzuela-Espinoza IG, ArmentaGálvez JM. Análisis de las propiedades físico-químicas y sensoriales de barra alimenticia a base de semillas y nueces sin componentes de origen animal. Rev Esp Nutr Hum Diet. 2020; 24(2): 143-53. doi: 10.14306/ renhyd.24.2.963

\section{InTRODUCción}

Las barras alimenticias surgen en el mercado hacia 1960, tras la popularización de barras diseñadas para los astronautas en sus viajes por el espacio exterior, estos productos para astronautas fueron la versión preliminar de las barras energéticas, diseñadas para cubrir los requerimientos energéticos ${ }^{1}$. Las barras energéticas pueden ser altamente nutritivas debido a que son una buena fuente de energía, ricas en fibra, proteína, vitaminas, antioxidantes, etc., y se preparan usando múltiples ingredientes como cereales, frutas, nueces, pasas, azúcar, etc. ${ }^{1}$ Son generalmente fortificadas usando una amplia variedad de proteínas como la soya o el trigo; fibras que incluyen salvado, avena, cebada; vitaminas, minerales, especies y otros nutrientes o ingredientes energéticos². Comercialmente existen diferentes tipos de barras: barras suplemento alimenticio, barras nutricionales para diabéticos, mujeres y niños, con alto contenido en proteína, alto contenido en fibra, ricas en vitaminas y minerales, barras con aditivos funcionales como probióticos ${ }^{3}$. Estos productos se han vuelto muy populares debido a los beneficios asociados con la salud. En encuestas hechas a los consumidores, se observa una tendencia a asociar a las barras como comida sana y de buen sabor, constituyéndose esto en una buena razón para su compra ${ }^{4}$. Interacciones físicas y químicas entre los ingredientes de la barra pueden ocurrir con el tiempo y afectar el sabor y la textura del producto. Mediciones de la actividad acuosa (aw) ayudan a predecir propiedades mecánicas, estabilidad y vida de anaquel ${ }^{5}$. Su textura está 
más relacionada con sus propiedades físicas y especialmente con las propiedades mecánicas. La mayoría de los alimentos procesados con características crujientes presentan una estructura porosa compuesta de agregados sólidos rodeados de aire ${ }^{6}$. Las propiedades mecánicas de estos agregados sólidos dependen de la composición y homogeneidad de los materiales y también de la cantidad y estructura de los poros. La no-uniformidad en la estructura interna de las barras crujientes resulta en un mecanismo muy complejo de fractura que involucra una deformación repetitiva de las subsecuentes capas que conforman la estructura. Esto genera un comportamiento mecánico irregular y una relación irreproducible de las fuerzas de deformación. Para mejorar las propiedades organolépticas y físicas de las barras suplementos, tales como sabor, aroma, apariencia y calidad nutricional existe la tendencia en la industria productora a incorporar mezclas de granos, semillas y/o combinación de concentrados de proteína de soya, suero de leche con salvado de cereales, avena y cebada7. Las nueces y semillas son fuentes ricas en lípidos y proteínas. Son ampliamente utilizadas por su contenido de aceites en mantequillas, barras alimenticias y también como alimento directo debido a sus características organolépticas $^{8}$. Del contenido de ácidos grasos comúnmente encontrados en semillas y nueces, el $70-80 \%$ son ácidos grasos poliinsaturados, los cuales son componentes de la membrana plasmática y de una lipoproteína encontrada en las células cerebrales conocida como lecitina. Por lo tanto, las semillas y nueces son excelentes nutrientes para el crecimiento de niños y bocadillos saludables para adultos ${ }^{8}$. Debido a su alto contenido en vitaminas $B$ y $E$, las nueces y semillas también presentan efectos de antienvejecimiento ${ }^{9}$. Cambios recientes en patrones de dietas y medidas para elevar la calidad de vida han sido asociados con incidencia en la obesidad, enfermedades cardiovasculares, diabetes, cáncer y otros tipos de enfermedades ${ }^{10}$. Estas enfermedades están relacionadas con los incrementos de los niveles de lípidos en plasma, trombosis, oxidación de lipoproteínas de baja densidad y con la formación de radicales libres. Estudios realizados en semillas y nueces han revelado que estos contienen polifenoles los cuales tienen efectos antioxidantes que previenen la ateroesclerosis, hipertensión y enfermedades cardiovasculares. También han mostrado tener efectos anticarcinógenos, antiinflamatorios, anti mutagénicos, antivirales y antialérgicos ${ }^{11}$. Estudios recientes presentados por la Asociación Americana del Corazón (AHA, por sus siglas en inglés) a partir de datos colectados a lo largo de 22 años afirman que dietas basadas en grasas monoinsaturadas a partir de plantas como las encontradas en aceites vegetales, aguacates, nueces y semillas están asociadas con riesgos bajos de mortalidad por enfermedades cardiacas y otras causas, esto si se compara con la ingesta de grasas monoinsaturadas provenientes de animales como las obtenidas de la carne, leche y huevos que están asociadas a riesgos más elevados de acuerdo a las investigaciones ${ }^{12}$. El objetivo de este trabajo se basó en la caracterización físicoquímica de una barra alimenticia elaborada a base de semillas y nueces libre de componentes de origen animal, su aceptación como un producto de colación (refrigerio) por una población seleccionada al azar de público atendiendo a un gimnasio de acondicionamiento físico y con alta actividad diaria y pruebas sensoriales efectuadas tanto instrumentalmente como por evaluadores semientrenados.

\section{$\longrightarrow$ MATERIAL Y MÉTODOS}

\section{Análisis Nutricional}

El material en estudio es una barra alimenticia, tipo agregado de semillas y nueces, de nueva creación que será lanzada al mercado como un producto alimenticio y orgánico sin componentes de origen animal. Los ingredientes de composición fueron: mezcla de nueces (almendra entera $48 \mathrm{~g}$, nuez de la India $30 \mathrm{~g}$, corazón de nuez pecana $15 \mathrm{~g}$, nuez pistache $12 \mathrm{~g}$ y nuez de Castilla 4g); mezcla de semillas (semilla de calabaza $8 \mathrm{~g}$, semilla de ajonjolí $8 \mathrm{~g}$, chía $4 \mathrm{~g}$ y linaza $4 \mathrm{~g}$ ), canela en polvo y coco tostado y molido $4 \mathrm{~g}$. El espectro de semillas y nueces fue seleccionado por ser los tipos de nueces y semillas más consumidas y abundantes en el mundo. También se incorporan los ingredientes de linaza en polvo, mantequilla de almendra, goma xantana hidratada, extracto de vainilla, cocoa, edulcorante ( $97 \%$ Eritritol, 3\% glucósido de esteviol). Un lote de 30 muestras de la formulación establecida como producto final por el fabricante se recibió en presentación individual con forma rectangular $(11 \mathrm{~cm} \times 5 \mathrm{~cm} \times 1,5 \mathrm{~cm})$ con un peso promedio de $46 \mathrm{~g}$ por porción para los análisis físico-químicos, textura, color y de vida útil. La cantidad de muestra necesaria para los análisis próximo y fisico-químico se obtuvo de la molienda y homogenización de las muestras individuales del producto. Para las pruebas de textura, color y vida útil se utilizó una barra individual del mismo lote por réplica en cada prueba.

\section{Análisis Próximo}

El análisis próximo incluyó contenido de humedad, contenido de fibra cruda, contenido de grasa y contenido de cenizas por métodos estandarizados de $\mathrm{AOAC}^{13}$. El contenido de proteína $(\% \mathrm{~N} \times 6,25)$ fue determinado por el Método Hach ${ }^{14}$. El contenido de carbohidratos totales se estimó como la diferencia de la suma total de otros análisis ${ }^{15}$. El análisis próximo se realizó en triplicado y se compararon los resultados. 


\section{Valor Energético}

El análisis de azúcares totales (monosacáridos libres y disacáridos) fue realizado por el método fenol-ácido sulfúrico ${ }^{16}$ y para los azúcares reductores (aldosas) se utilizó el método del ácido dinitrosalicílico (DNS) ${ }^{17}$. El valor energético se calculó en base a la composición proximal: porcentaje de proteínas, grasas y carbohidratos, utilizando los factores de Atwater (Proteínas: $4 \mathrm{kcal} / \mathrm{g}$ - Grasas: $9 \mathrm{kcal} / \mathrm{g}$ - Carbohidratos: 4kcal/g)

\section{Calidad de Lípidos}

La materia grasa extraída de la cuantificación del contenido de lípidos se derivatizó a metil-ésteres (FAMEs) por metilación con ácido clorhídrico metanólico 3M para la determinación del perfil de ácidos grasos ${ }^{18}$. Este análisis se realizó mediante cromatografía de gases en un Cromatógrafo Thermo Fisher Scientific Focus equipado con un detector de ionización de flama (FID) y con una columna capilar D-B23 para FAMEs. El régimen de temperatura de la columna fue como sigue: temperatura inicial de horno $80^{\circ} \mathrm{C}$ (sostenida por 2 minutos) hasta alcanzar $240^{\circ} \mathrm{C}$ a velocidad de $10^{\circ} \mathrm{C} /$ minuto (sostenida por 5 minutos). Las temperaturas del inyector y del detector fueron de 225 y $250^{\circ} \mathrm{C}$, respectivamente. Los FAMEs se identificaron comparando sus tiempos de retención con los de la mezcla de estándares usada como estándar de calibración (FAME Mix GLC - 10, SUPELCO). Para el cálculo, los ácidos grasos se normalizaron hasta el $100 \%$, considerando la composición (\% en peso) de los datos de composición de ácidos grasos (\% de área).

\section{Determinación de Minerales}

La determinación de minerales esenciales como sodio $(\mathrm{Na})$, potasio $(\mathrm{K})$, magnesio $(\mathrm{Mg})$, calcio $(\mathrm{Ca})$, hierro $(\mathrm{Fe})$, zinc $(\mathrm{Zn})$, manganeso (Mn) y cobre (Cu) se realizó por el método Espectrofotometría de Absorción Atómica $(E A A)^{19}$. Muestras de barra molida de $3 \mathrm{~g}$ fueron secadas en horno a temperatura de $105^{\circ} \mathrm{C}$ por 12 horas. Posteriormente las muestras se sometieron a mineralización combinada en mufla usando peróxido de hidrógeno como oxidante: las muestras fueron mineralizadas en seco a $450^{\circ} \mathrm{C}$ por 12 horas. A las cenizas resultantes se les agregaron $2 \mathrm{~mL}$ de peróxido de hidrógeno, se vaporizaron a sequedad bajo campana de extracción de vapores y recalcinadas por 12 horas a $450^{\circ} \mathrm{C}$, este procedimiento se repitió cuatro veces. Las cenizas blancas resultantes fueron disueltas en $\mathrm{HNO}_{3} 1 \mathrm{M}$ para su análisis por EAA en un Equipo GBC, Avanta, usando lámparas multielementos de cátodo hueco de los metales a analizarse. Se construyeron curvas de calibración con soluciones estándares de cada elemento. Para evaluar las características analíticas del método para cada elemento, los límites de detección fueron calculados de acuerdo con las re- glas IUPAC ${ }^{20}$. La exactitud del método se confirmó agregando cantidades conocidas del analito a tres diferentes muestras y procesando las mezclas como se ha descrito.

\section{Vida Útil de la Barra Alimenticia}

Índice o Valor de peróxido (VP): Para determinar el nivel de durabilidad del producto, se determinó el índice de peróxido por el método de la Norma Mexicana ${ }^{21}$ NMX-F-154. Las muestras fueron sometidas a las condiciones menos favorables de almacenamiento (temperatura de $40^{\circ} \mathrm{C}$ durante 15 días). El análisis consistió en disolver los lípidos de una muestra de $5 \mathrm{~g}$ en una solución de ácido acético glacial-isooctano (3:2 $\mathrm{v} / \mathrm{v})$. Posteriormente se adicionó un exceso de solución de ioduro de potasio, el cual reacciona con los peróxidos, que transforman los iones ioduro en iodo molecular. La mezcla es valorada con solución de tiosulfato de sodio estandarizada, usando como indicador solución de almidón. El índice de peróxido se calculó como lo indica la ec. 1.

$$
\text { Índice de Peróxido }=\frac{(S-B)}{W} \times 1.000 \quad \text { Ec. (1) }
$$

Donde:

Índice de peróxido: $\mathrm{mEq}$ de peróxido por $\mathrm{kg}$ de muestra; $\mathrm{S}$ : volumen de valorante gastado $(\mathrm{mL})$ por muestra; $B$ : volumen de valorante gastado $(\mathrm{mL})$ por blanco; $\mathrm{N}$ : normalidad de solución valorante de $\mathrm{Na}_{2} \mathrm{~S}_{2} \mathrm{O}_{3}(\mathrm{mEq} / \mathrm{mL})$; 1.000 : conversión de unidades $(\mathrm{g} / \mathrm{kg})$; W: peso de muestra $(\mathrm{g})$

Actividad del agua: La mínima actividad acuosa a la cual los microorganismos pueden crecer es 0,60 (actividad del agua por debajo de la cual las actividades fisiológicas necesarias para la división celular no se cumplen) 22. La actividad acuosa se determinó usando un equipo AQUA LAB 4 TEV (Decagon Devices, USA). Las muestras fueron molidas para su medición en las copas del equipo y se consideró que alcanzaron el equilibrio cuando la actividad acuosa se mantuvo por 1 minuto.

\section{Pruebas de Textura y Color de la Barra Alimenticia}

Medición de la Textura: El análisis de la textura es un excelente procedimiento instrumental, que ayuda a medir y a cuantificar parámetros tales como: dureza, gomosidad, elasticidad y cohesividad, entre otros, que se relacionan a su vez con variables como la tasa de deformación aplicada y la composición del producto. El método instrumental usado en este trabajo fue la prueba de flexión de tres puntos, que es un método destructivo, que puede imitar el proceso de masticación, pero en el cual no hay relación con las sensaciones involucradas en la boca ${ }^{23}$. El equipo usado para las pruebas de textura fue 
un Texturómetro AMETEK Brookfield CT3 - 1500 (Middleboro, MA, USA) con Probeta de Flexión de tres puntos y operado por Software TexturePro CT V1.2; provisto de una plataforma de aluminio, sobre la cual se colocaron las muestras. La velocidad del proceso fue determinada mediante ensayos previos y se fijó en $2 \mathrm{~mm} / \mathrm{s}$. Se determinó el cambio en la dureza del producto en un período de 30 días con mediciones al inicio y posteriormente cada 10 días cuando las barras permanecieron a temperatura ambiente $\left(22^{\circ} \mathrm{C}-25^{\circ} \mathrm{C}\right)$.

Medición del Color: Existen una variedad de sistemas que se han desarrollado para describir los colores, entre estos el más común en la búsqueda del control de calidad de los alimentos es el CIE L*a*b*24. En este sistema, el espacio del parámetro $L^{*}$ es la brillantez y varía de negro $\left(L^{*}=0\right)$ a blanco $\left(L^{*}=100\right)$ y los parámetros de los colores complementarios $\mathrm{a}^{*} \mathrm{y} \mathrm{b}^{*}$, con $a^{*}$ representando al color verde cuando su valor es negativo y al color rojo cuando positivo y con b* representando al color azul cuando su valor es negativo y amarillo cuando positivo. Ambos parámetros varían en el rango de $-60 \leq a^{*}, b^{*} \leq+60$. Para la medición del color se utilizó un espectrofotómetro HunterLab MiniScan EZ 4500 L (Hunter Associates Laboratory Inc., Reston, VA, USA). Las determinaciones se hicieron en ambas superficies de las barras (lados superior, inferior y laterales), un total de 3 réplicas por muestra.

\section{Análisis Sensorial}

El método de análisis sensorial utilizado en este trabajo fue la prueba de aceptación con escala hedónica. Esta es una prueba que mide la aceptación subjetiva y preferencia del producto por parte del consumidor. Este tipo de prueba consiste en dar muestras del producto a los catadores y cuestionarlos a través de encuesta acerca de sus observaciones de acuerdo con una escala establecida. En la escala hedónica, el catador expresa su aceptación por el producto siguiendo una escala previamente establecida, la cual varía gradualmente con la intensidad de sus atributos ${ }^{25}$. La población de catadores estuvo compuesta de un total de 75 participantes, todos ellos asistentes asiduos a gimnasios de condición física, con edades entre los 18 y 61 años, distribuidos como sigue: $48 \%$ menores de 25 años, 34\% entre 25 y 35 años, 15\% entre 36 y 45 años y el $3 \%$ fueron mayores de 45 años. Considerando el género, $47 \%$ fueron hombres y $53 \%$ fueron mujeres. Los encuestados realizaron la evaluación respondiendo un cuestionario de acuerdo con los atributos a evaluar: aspecto, color, olor y sabor en una escala hedónica de nueve puntos en el nivel de aceptabilidad, donde 1 denota el valor mínimo (extremadamente desagradable) y 9 (extremadamente agradable). Los datos de las encuestas se trataron en software MINITAB 17 y Excel.

\section{Análisis Estadístico}

Las pruebas físico-químicas se realizaron por triplicado a menos que se indicara de otra manera. Los resultados obtenidos fueron estadísticamente analizados para el análisis de varianza (ANOVA) y la prueba de medias (Tukey) con un $5 \%$ de nivel de significancia para cada prueba.

\section{RESULTADOS}

\section{Análisis Próximo}

La composición química proximal del producto se presenta en la Tabla 1. Como era de esperarse, la barra alimenticia presentó un bajo contenido de humedad (3,49\%); disminuyendo así la probabilidad de crecimiento microbiano, fermentaciones imprevistas, germinación prematura en semillas y muchos

Tabla 1. Análisis proximal de barra alimenticia a base de semillas y nueces.

\begin{tabular}{l|c|c|c} 
Determinación & Método & $\% \mathrm{p} / \mathrm{p}$ & Norma \\
\hline Humedad & Balanza de Humedad por IR & $3,49 \pm 0,06$ & NMX-F-428-1982 \\
\hline Cenizas & Calcinación & $3,59 \pm 0,74$ & NMX-F-066-S-1978 \\
\hline Grasas y Aceites & Extracción Soxhlet & $46,96 \pm 3,71$ & NMX-F-615-2004 \\
\hline Proteína & $\mathrm{HACH}$ & $25,68 \pm 0,40$ & Método HACH \\
\hline Azúcares Totales & Fenol $-\mathrm{H}_{2} \mathrm{SO}_{4}$ & $4,39 \pm 0,07$ & Nielsen-1998 \\
\hline Azúcares Reductores & Método DNS & $2,11 \pm 0,02$ & NMX-F-312-1978 \\
\hline Fibra Cruda & Método Weende & $7,91 \pm 0,60$ & NMX-F-090-S-1978 \\
\hline Carbohidratos & Empírico & $12,37 \pm 0,94$ & FAO \\
\hline
\end{tabular}


otros cambios bioquímicos indeseables normalmente asociados con la presencia de humedad.

El contenido de cenizas (3,59\%) es consistente con lo reportado por Venkatachalam y este valor tan elevado es indicativo del alto contenido mineral en semillas y nueces ${ }^{26}$. Tanto el alto contenido proteico $(25,68 \%)$ como el alto contenido de lípidos $(46,96 \%)$ de la barra alimenticia se deben al contenido de mezclas de semillas (calabaza, girasol, ajonjolí, linaza, chía) y de nueces (almendra, nuez de la India, pistache, nuez de Castilla y nuez pecana) en la formulación de la barra ${ }^{26}$. El contenido de azúcares totales y azúcares reductores (4,39\% y $2,11 \%$, respectivamente) es consistente con los valores reportados por la literatura de contenidos de azúcares en semillas y nueces los cuales se encuentran muy por debajo de los azúcares aportados por las barras elaboradas a base de cereales, $23,8 \%$ 27. El contenido de fibra cruda de la barra alimenticia de $7,91 \%$ (3,7g por porción) se encuentra por debajo de la ingesta diaria recomendada por la FDA de $25 \mathrm{~g}$ basada en una dieta de 2.000 calorías diarias ${ }^{28}$, y donde una sola barra aportaría el $15 \%$ aproximadamente, de la fibra diaria requerida.

\section{Valor energético y Composición de ácidos grasos}

La barra alimenticia presentó un buen aporte calórico, con un valor energético de 574,84 calorías por cada $100 \mathrm{~g}$ de muestra calculado por el método Atwater ${ }^{15}$. Los resultados obtenidos del perfil de ácidos grasos que componen los aceites extraídos de la barra alimenticia mostraron que los ácidos grasos más abundantes presentes fueron: ácido palmítico (C16:0), ácido esteárico (C18:0), ácido oleico (C18:1), ácido linoleico (C18:2) y ácido linolénico (C18:3) con valores de 14,6; 4,5; 58,0; 13,6 y $9,3 \% \mathrm{p} / \mathrm{p}$ respectivamente. Se observa una proporción de $19 \%$ de ácidos grasos saturados y $81 \%$ de ácidos grasos insaturados. El contenido del ácido palmítico (C16:0) en el aceite extraído del alimento, debe su aportación a los ingredientes de semilla de calabaza y coco molido en la formulación. El contenido de ácido esteárico (C18:0) proviene principalmente de pistachos, semillas de girasol y de las almendras mientras que los ácidos grasos, oleico (C18:1), linoleico (C18:2) y linolénico C18:3) son mayormente aportación de la nuez de nogal. Independientemente del tipo de semilla o nuez, el ácido oleico (C18:1) y el ácido linoleico (C18:2) son los contribuyentes predominantes al contenido de lípidos en semillas y nueces ${ }^{26}$. Se hizo una estimación empírica del contenido de los ácidos grasos omega-6 y omega-3 en el producto. El método utilizado fue el Método de aportaciones basado en la aportación de los ácidos grasos de cada componente en la formulación y en base a las concentraciones de los omega- 6 y omega- 3 reportados en la literatura de las nueces y semillas utilizadas en la preparación de la barra ${ }^{29}$. El resultado obtenido mostró un contenido de $5,8 \mathrm{~g}$ de omega- 6 y $0,61 \mathrm{~g}$ de omega-3 por porción de barra alimenticia (aproximadamente $46 \mathrm{~g} /$ porción). La dosis diaria recomendada de omega- 3 es de $1,6 \mathrm{~g}$ y $1,1 \mathrm{~g}$ para hombres y mujeres respectivamente, y de omega- 6 es de $17 \mathrm{~g}$ para hombres y $12 \mathrm{~g}$ para mujeres ${ }^{30,31}$

\section{Elementos Esenciales}

Los minerales esenciales en la barra alimenticia se determinaron por Espectrofotometría de Absorción Atómica (EAA) de acuerdo con las condiciones de operación establecidas en el apartado de Material y Métodos. Los minerales actúan en el cuerpo humano como cofactores de las reacciones biológicas enzimáticas y mantienen en balance el pH corporal. También, los minerales facilitan la transferencia de nutrientes a través de la membrana celular, entre otras importantes funciones. Existen dos categorías de minerales esenciales para el cuerpo humano, macrominerales y microminerales ${ }^{32}$. La Tabla 2 muestra los resultados obtenidos del contenido de minerales esenciales determinados en la barra alimenticia caracterizada en este trabajo y los requerimientos diarios. De estos resultados se observa que una porción de la barra alimenticia aporta casi en su totalidad los microminerales de cobre, hierro, manganeso

Tabla 2. Valores de minerales esenciales determinados en barra alimenticia a base de semillas y nueces.

\begin{tabular}{|c|c|c|c|c|c|}
\hline \multicolumn{3}{|c|}{ Macrominerales } & \multicolumn{3}{|c|}{ Microminerales } \\
\hline Nutriente & $\begin{array}{l}\text { Barra Nutritiva } \\
\text { (mg/porción) }\end{array}$ & $\begin{array}{l}\text { Valor diario* } \\
(\mathrm{mg} / \mathrm{d})\end{array}$ & Nutriente & $\begin{array}{l}\text { Barra nutritiva } \\
\text { (mg/porción) }\end{array}$ & $\begin{array}{l}\text { Valor diario* } \\
\text { (mg/d) }\end{array}$ \\
\hline Calcio & $304,00 \pm 7,30$ & $1.000,00$ & Cobre & $0,28 \pm 0,01$ & 0,90 \\
\hline Magnesio & $110,00 \pm 2,20$ & 350,00 & Hierro & $10,80 \pm 0,19$ & 8,00 \\
\hline Potasio & $46,00 \pm 0,96$ & 3,50 & Manganeso & $1,41 \pm 0,03$ & 2,00 \\
\hline Sodio & $42,00 \pm 0,90$ & 2,40 & Zinc & $6,00 \pm 0,12$ & 8,00 \\
\hline
\end{tabular}

*Valores recomendados $\geq 19$ años $^{33}$. Resultados promedio del análisis de 3 réplicas. 
y zinc diarios recomendados. En cuanto a los macrominerales, sólo en potasio y sodio se ve una deficiencia marcada.

\section{Vida Útil de la Barra Alimenticia a base de semillas y nueces}

Velocidad de rancidez: La oxidación de las grasas y aceites contenidos en los alimentos es un importante indicador de la vida en anaquel de los alimentos. Los radicales libres de peróxido reaccionan con ácidos grasos insaturados para formar hidroperóxidos y radicales libres de alquilo (propagación). Estos productos no tienen olor ni sabor. La reacción continúa hasta que dos radicales inestables reaccionan entre sí (terminación) ${ }^{34}$. El parámetro que se utilizó en este trabajo para medir el deterioro de la barra alimenticia a base de semillas y nueces fue el VP, el cual determina las concentraciones de los hidroperóxidos formados en la fase inicial del proceso de oxidación de las grasas y lípidos. La Figura 1 muestra los resultados obtenidos de la medición de V.P. en una simulación del proceso de oxidación bajo las condiciones menos favorables de almacenamiento del producto para determinar la vida de anaquel (15 días a $40^{\circ} \mathrm{C}$, en oscuridad).

Los cambios registrados en la medición del valor de peróxidos durante el almacenamiento de la barra muestran valores

Figura 1. Perfil del valor de peróxido (VP) de barra almacenada $40^{\circ} \mathrm{C}, 15$ días, $n=3$.

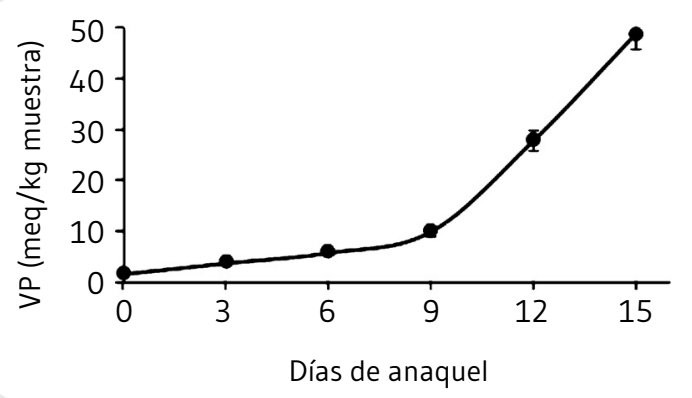

bajos de oxidación (10meq/kg de muestra) hasta los 9 días en anaquel bajo las condiciones establecidas. La Organización de las Naciones Unidas para la Alimentación y la Agricultura (FAO, por sus siglas en inglés), establece un límite máximo permitido de $10 \mathrm{meq} / \mathrm{kg}$ para el valor de peróxidos en alimentos ${ }^{35}$. Este comportamiento retardado de oxidación en los primeros 9 días es muy probable que se deba a la presencia de antioxidantes naturales presentes en las semillas y nueces como polifenoles, los cuales actúan como donadores de átomos de hidrógeno a los radicales libres formados durante la fase de iniciación del proceso de oxidación ${ }^{36}$.

\section{Actividad del agua (aw)}

La actividad acuosa se determinó en las mismas muestras de las barras alimenticias que fueron sometidas a un período de 15 días de almacenamiento a $40^{\circ} \mathrm{C}$ en la oscuridad y empacadas para la prueba de rancidez. Los resultados mostraron que durante el periodo de almacenamiento la actividad acuosa permaneció entre 0,37 y 0,50. En este rango de actividades acuosas, se considera que no es de esperar crecimiento microbiano. Una actividad acuosa de 0,6 es el punto crítico al cual pudiera haber crecimiento microbiológico si el contenido de humedad incrementara significativamente. Un incremento en la actividad acuosa por arriba de 0,8 incrementaría las posibilidades de crecimiento microbiano ${ }^{37}$.

\section{Pruebas de Textura y Color}

Dureza de la barra: La determinación de dureza se realizó en un texturómetro CT3 - 1500 V 3.0, marca Brookfield, operando con el software TexturePro. Modo de operación: medición de fuerza por compresión, probeta de flexión de tres puntos TA - TPB con sonda TA7 y carga de celda de $1.500 \mathrm{~g}$ bajo las siguientes condiciones: velocidades de pre-prueba y prueba: $2 \mathrm{~mm} / \mathrm{s}$ y $0,1 \mathrm{~mm} / \mathrm{s}$, respectivamente; distancia de $50 \%$; 3 réplicas $^{38}$. Las dimensiones promedio de las barras de prueba fueron: longitud $110 \mathrm{~mm}$, ancho $50 \mathrm{~mm}$ y espesor $10 \mathrm{~mm}$. La Tabla 3 muestra los resultados obtenidos en la determinación de dureza de la barra alimenticia con respecto al tiempo

Tabla 3. Perfil de textura de la barra alimenticia en relación con el tiempo de almacenamiento a temperatura ambiente.
Parámetros
0
10
20
30
Fracturabilidad (g)
$229,20 \pm 1,70$
$446,30 \pm 2,10$
$859,60 \pm 3,70$
Deformación por dureza (mm)
$3,05 \pm 0,05$
$3,12 \pm 0,07$
$3,28 \pm 0,08$
$1.530,20 \pm 6,80$
Deformación por dureza (\%)
$2,80 \pm 0,02$
$2,8 \pm 0,02$
$3,0 \pm 0,02$
$3,46 \pm 0,10$
Trabajo terminado (m])
$11,34 \pm 0,32$
$21,53 \pm 0,91$
$40,01 \pm 1,76$
$3,1 \pm 0,02$
$60,09 \pm 2,09$ 
de almacenamiento a temperatura ambiente $\left(22^{\circ} \mathrm{C}-25^{\circ} \mathrm{C}\right)$ en anaquel. Se determinó que un rango de tiempo de 30 días de almacenamiento afectaba la dureza de las barras.

\section{Color}

El color es un parámetro importante en barras nutritivas comerciales. Los resultados de las mediciones de color de la barra alimenticia en este trabajo para diferente tiempo de almacenamiento (hasta 15 días), en términos de los valores Hunter de $L^{*}, a^{*}$ y $b^{*}$, se presentan en la Figura 2. Del análisis de los resultados en las pruebas de color se observa que el producto presenta aspecto algo brillante y con una mayor tendencia hacia los tonos rojizos-amarillos. Para las muestras de barras almacenadas por 15 días a temperatura ambiente, el análisis de varianza (ANOVA) muestra valores $p>0,05$ para las coordenadas de color $L^{*}, a^{*} \& b^{*}$, por lo que se establece que no hay diferencia estadística marcada entre los valores de color medidos en las muestras, por lo tanto, el almacenamiento no presenta un efecto marcado en el color del producto.

\section{Pruebas sensoriales de aceptación}

El análisis sensorial se realizó principalmente para relacionar la barra alimenticia a base de semillas y nueces con una alta aceptabilidad y contribuir con la información nutritiva en el conocimiento del producto selecto. En este estudio se encontró que la presencia de nueces y semillas se percibió significativamente en el producto. El ANOVA se realizó para la prueba de aceptación del producto por una población asistente a gimnasios teniendo como efectos principales para producto y consumidor ${ }^{25}$. Los análisis de datos se realizaron usando el software Minitab versión 16. La evaluación sensorial de aceptación del producto indicó que la barra alimenticia fue bien aceptada por los consumidores para

Figura 2. Perfil de color en barras nutritivas en 15 días de almacenamiento.

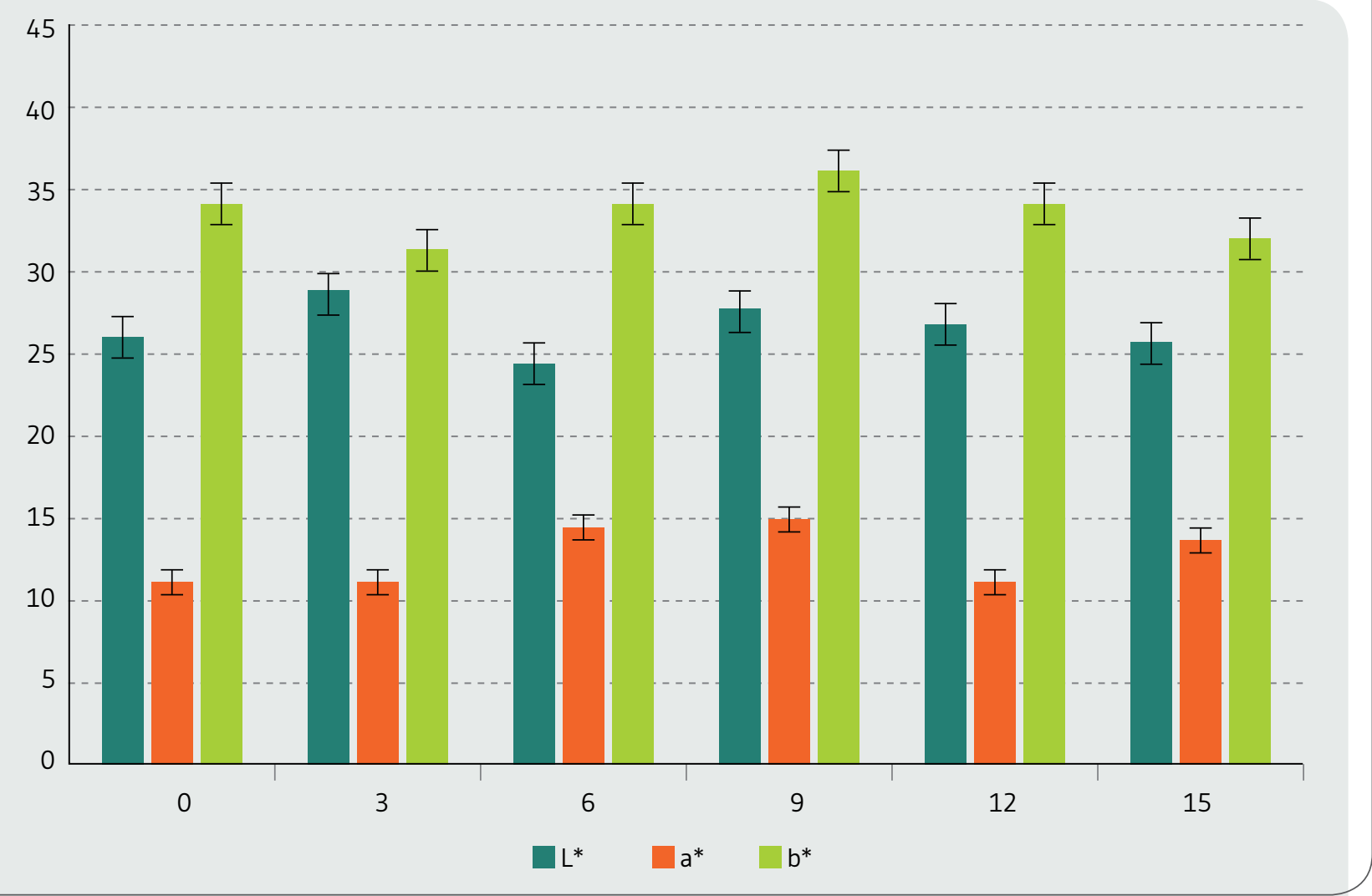

L*: designa la brillantez de la muestra; $\mathbf{1 0 = b l a n c o ; ~} \mathbf{0 = n e g r o ; ~} \mathbf{a}^{*}$ : indica tonos rojizos cuando los valores son positivos y tonos verdes cuando los valores son negativos; b*: indica tonos amarillos cuando los valores son positivos y azules cuando los valores son negativos. 
todos los atributos evaluados (aspecto, color, olor y sabor). La Figura $3 \mathrm{~A}$ muestra el análisis de los resultados de la prueba sensorial realizada y se observa que los valores asignados por los consumidores (75) se inclinan hacia el rango de 6 a 9. En la escala hedónica el valor 6 corresponde a "me gusta ligeramente" y el valor hedónico 9 corresponde a "me gusta en extremo".

Las variantes de los valores en los atributos del producto no cayeron en la categoría de extremo desagradable. Los porcentajes de aceptación de cada atributo por la población en el rango de valores de 7 a 9 de la escala hedónica para la barra alimenticia están representados en la Figura 3B. Este estudio mostró que el sabor y el olor se impusieron sobre el color y aspecto del producto.

\section{Discusión}

El análisis químico proximal, destaca el alto contenido de proteína (26\%), lo cual puede ser una alternativa como fuente de proteína en la elaboración de alimentos tipo colación a base de nueces y semillas. También el contenido en lípidos (47\%) se encuentra entre los valores más altos del análisis químico, que por la formulación de la barra alimenticia tiene su origen en semillas y nueces con una alta aportación en ácidos grasos omega-3 y omega-6 (US. Department of Health \& Human Services). Los ácidos grasos esenciales son los ácidos grasos comúnmente encontrados en nueces y semillas (70-80\%), los cuales son componentes de la membrana plasmática y contienen una lipoproteína encontrada en cé- lulas del cerebro conocida como lecitina ${ }^{39}$. Los resultados de humedad $4 \%$, carbohidratos $13 \%$, fibra cruda $8 \%$ y cenizas $4 \%$ están dentro de lo reportado en otras fuentes bibliográficas con valores similares. La fibra cruda tiene varias propiedades funcionales como facilitar funciones alimenticias, procesos de reabsorción de compuestos no deseables como el colesterol, reducir tiempo de tránsito intestinal, aumenta la estabilidad de alimentos modificando su estructura, densidad y textura ${ }^{3}$. La barra energética mostró ser una buena fuente de calcio, magnesio, hierro, manganeso y zinc y sus valores fueron mayores o cercanos al valor diario recomendado $^{33}$. La actividad acuosa ( $a w$ ) en este estudio fue adecuada $(0,3-0,5)$ ya que se relaciona con la conservación de cualquier producto deshidratado o seco. La aw ayuda a predecir la estabilidad y vida útil de los alimentos, también a establecer cualidades nutricionales y organolépticas, así como otros puntos relacionados con la inocuidad y calidad de los alimentos ${ }^{36}$. ljabadeniyi y Col. ${ }^{40}$ mencionan que patógenos Gram negativo sobrevivieron en nueces y chocolates con aw tan bajas como 0,648. El VP es una medida de la concentración de los productos primarios de la oxidación lipídica utilizado en la determinación de la velocidad de deterioro de alimentos con alto contenido en grasas y aceites. Los resultados aquí reportados del VP con respecto al tiempo de almacenamiento coinciden con otros estudios realizados a nueces y semillas ${ }^{41}$.

En las evaluaciones sensoriales, los atributos mejor calificados fueron el olor y el sabor y las menores preferencias se dieron para el color y apariencia. Los valores obtenidos de las evaluaciones sensoriales de aceptabilidad en barras fueron más altos que aquellos obtenidos por otros reportes de barras alimenticias ${ }^{42}$.

Figura 3. Análisis de los resultados de la prueba sensorial.

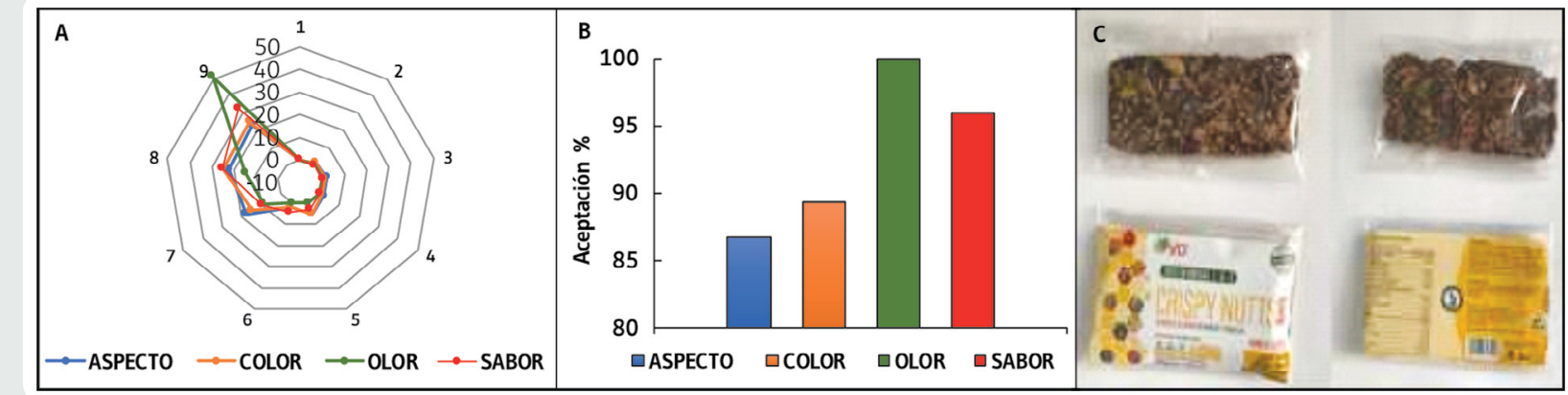

(A) Evaluación de: Aspecto, Color, Olor y Sabor de la barra nutritiva por una población de 75 consumidores. Escala hedónica de 1-9, siendo 1=Extremo desagradable y $9=$ =xtremo agradable. (B) Porcentajes de población de consumidores evaluando barra en rango de valores de escala hedónica 7-9 para los atributos de Aspecto, Color, Olor y Sabor que corresponde a "me gusta ligeramente - me gusta en extremo" contra su propio control; de acuerdo con el ANOVA ( $p>0,05$ ). (C) Presentación del producto sin etiquetado para su evaluación sensorial de aceptación y su presentación final de etiquetado para su lanzamiento al mercado. 


\section{CONCLUSIONES}

Los resultados del análisis próximo revelaron un producto alimenticio con alto contenido proteico (25\%) y lipídico (46\%) sin afectar negativamente el contenido de fibra cruda, minerales esenciales o sus atributos sensoriales. Las pruebas sensoriales demostraron su genuina aceptación entre una población demandante de alimentos nutricionales de rápida disponibilidad, fácil manejo y térmicamente estables. Este estudio indica que el producto analizado tiene las posibilidades de ser un alimento comercializable por su aporte nutrimental y sus atributos sensoriales.

\section{AGRADECIMIENTOS}

Al Consejo Nacional de Ciencia y Tecnología de México por el recurso otorgado para la realización de este trabajo.

\section{CONFLICTO DE INTERESES}

Los autores expresan que no existen conflictos de interés al redactar el manuscrito.

\section{REFERENCIAS}

(1) Warren RM, Best Energy Bars: Crunchy, Chewy, Tasty... and Healthy, Too? [Internet]. CR Consumer Reports Organization. 2018 [cited 15 February 2019]. Available from: https://www. consumerreports.org/snack-bars/best-energy-bars-reviewcrunchy-chewy-tasty-healthy/

(2) Narang P. The Truth about Energy Bars: Are they Really Good for You? [Internet]. NDTV FOOD. 2017 [cited 16 February 2019]. Available from: https://food.ndtv.com/food-drinks/the-truthabout-energy-bars-are-they-really-good-for-you-1208551

(3) Kavitha B, Vijayalakshmi R, Poorna CR, Yalagala IM, \& Sugasini D. Nutritional Evaluation and Cell Viability of Formulated Probiotic Millet Fruit Bar. J Food Nutr Disor, 2018; 7(2). doi: 10.4172/2324-9323.100024H6

(4) Weber J, Callaghan M. Optimizing ready-to-use therapeutic foods for protein quality, cost, and acceptability. Food Nutr Bull. 2016; 37(Suppl 1): S37-S46.

(5) Jakubczyk E, Marzec A, Lewicki PP. Relationship between water activity of crisp bread and its mechanical properties and structure. Pol. J. Food Nutr. Sci. 2008; 58(1): 45-51.
(6) Luyten H, Plijter JJ, Van Vliet T. Crispy/crunchy crusts of cellular solid foods: a literature review with discussion. J Texture Stud. 2005; 35(5): 445-92.

(7) Indrani D, Soumya C, Rajiv J, Rao G V. Multigrain bread-its dough rheology, microstructure, quality and nutritional characteristics. J Texture Stud. 2010; 41(3): 302-19.

(8) Venkatachalam M, Sathe SK. Chemical composition of selected edible nut seeds. J. Agric Food chem. 2006; 54(13): 4705-14.

(9) McClees H, Raw Nuts and Seeds That Support Collagen Production in the Body [Internet]. One Green Planet-HEALTH. 2019 [cited 16 October 2019]. Available from: https://www. onegreenplanet.org/natural-health/raw-nuts-and-seeds-thatsupport-collagen-production-in-the-body/

(10) Moon HK, Lee HJ, Park Y. Comparison of health status and dietary habits by percent body fat (PBF) change for adult women in the weight control program by the community health center. Korean J Community Nutr. 2007; 12(4): 477-88.

(11) Pincemail J. Free radicals and antioxidants in human diseases. In Analysis of free radicals in biological systems. Switzerland, Birkhäuser Basel; 1995, p.83-98.

(12) Ducharme J. Plant Fats are Healthier Than Animal Fats, Study Says [Internet]. Health-Diet/Nutrition-TIME. 2019 [cited 16 October 2019]. Available from: https://time.com/5208817/ plant-animal-fats/

(13) Association of Official Agricultural Chemists. AOAC. Vol 2. 18 th ed. Gaithersburg, MD., AOAC International. Vol 2. 18th ed. AOAC International print, 2005.

(14) Rossi AM, Villarreal M, Juárez MD, Sammán NC. Nitrogen contents in food: A comparison between the Kjeldahl and Hach methods. An Asoc Quím Argent. 2004; 92(4-6): 99-108.

(15) Maclean W, Harnly J, Chen J, Chevassus-Agnes S, Gilani G, Livesey $G$, Warwick P. Food energy-Methods of analysis and conversion factors. In Food and Agriculture Organization of the United Nations, Technical Workshop Report, Rome. 2003; Vol. 77.

(16) Nielsen SS. Food analysis. 4th ed. New York (USA): Springer Science; c2010. Chapter 10, Carbohydrate Analysis; p. 147-154.

(17) Miller G L, Blum R, Glennon W E, Burton A L. Measurement of carboxymethylcellulase activity. Anal Biochem. 1960; 1(2): 127-132.

(18) Aldai N, Murray BE, Nájera Al, Troy DJ, Osoro K. Derivatization of fatty acids and its application for conjugated linoleic acid studies in ruminant meat lipids. J Sci Food Agric. 2005; 85(7): 1073-83.

(19) Winiarska-Mieczan A, Kwiecien M, Kwiatkowska K, Krusinski R. Breakfast cereal as a source of sodium, potassium, calcium and magnesium for school-age children. J Elem. 2016; 21(2): 571-84.

(20) Long GL, Winefordner JD. Limit of detection. A closer look at the IUPAC definition. Anal Chem. 1983; 55(7): 712A-724A.

(21) NMX-F-154-SCFI-2010. Alimentos - Aceites y Grasas Vegetales o Animales. Determinación del Valor de Peróxido - Método de Prueba. Foods - Vegetable or Animal Fats and Oils. Peroxide Value Determination. In: Normas Mexicanas. Dirección General de Normas, editor. México, D.F.: Secretaría de Economía; 2011.

(22) Beuchat LR, Komitopoulou E, Beckers H, Betts RP, Bourdichon $F$, Fanning $S$, et al. Low-water activity foods: increased concern as vehicles of foodborne pathogens. JFP. 2013; 76(1): 150-72. 
(23) Bland JM, Bett-Garber KL, Li CH, Brashear SS, Lea JM, Bechtel PJ. Comparison of sensory and instrumental methods for the analysis of texture of cooked individually quick frozen and fresh-frozen catfish fillets. Food Sci Nutr. 2018; 6(6): 1692-705.

(24) Pattee HE, Giesbrecht FG, Young CT. Comparison of peanut butter color determination by CIELAB L*, $\mathrm{a}^{*}, \mathrm{~b}^{*}$ and Hunter color-difference methods and the relationship of roasted peanut color to roasted peanut flavor response. ] Agric Food Chem. 1991; 39(3): 519-23.

(25) Cordero-Bueso GA. Análisis Sensorial de los Alimentos. 1st ed. Madrid (España): AMV Ediciones. c2017. Chapter 4, Tipos de pruebas de análisis sensorial; p. 155-182.

(26) Venkatachalam M, Sathe, SK. Chemical composition of selected edible nut seeds. Journal of agricultural and food chemistry. 2006; 54(13): 4705-14.

(27) Zupanič N, Miklavec K, Kušar A, Žmitek K, Fidler Mis, N, Pravst I. Total and Free Sugar Content of Pre-Packaged Foods and Non-Alcoholic Beverages in Slovenia. Nutrients. 2018; 10(2): E151.

(28) United States. Food \& Drug Administration. Department of Health and Human Services. Dietary Fiber [Document on the Internet]; 2018 [cited 12 October 2018]. Available from: https://www.accessdata.fda.gov/scripts/ interactivenutritionfactslabel/factsheets/Dietary_Fiber.pdf

(29) Drenjančevič I, Kralik G, Kralik Z, Mihalj M, Stupin A, Novak $S$, Grčevič M. The Effect of Dietary Intake of Omega-3 Polyunsaturated Fatty Acids on Cardiovascular Health: Revealing Potentials of Functional Food. In: Naofumi S, Viduranga $W$, eds. Superfood and Functional Food. InTech; 2017. p. 207-32.

(30) United States. Department of Health \& Human Services. National Institutes of Health. Omega-3 and Omega-6 Fatty Acids [Fact Sheet for Health Professionals]; 2018 [cited 12 October 2018]. Available from: https://ods.od.nih.gov/ factsheets/Omega3FattyAcids-HealthProfessional/

(31) Sheppard KW, Cheatham CL. Omega-6/omega-3 fatty acid intake of children and older adults in the US: Dietary intake in comparison to current dietary recommendations and the Healthy Eating Index. Lipids Health Dis. 2018; 17(1): 43.

(32) Martínez-Ballesta MC, Dominguez-Perles R, Moreno DA, Muries B, Alcaraz-López C, Bastías E. Minerals in plant food: effect of agricultural practices and role in human health. A review. Agron Sustain Dev. 2010; 30(2): 295-309.
(33) United States. Department of Health \& Human Services. National Institutes of Health. Nutrient Recommendations: Dietary Reference Intake [Health Information]; 2018 [cited 12 October 2018]. Available from: https://ods.od.nih.gov/Health_ Information/Dietary_Reference_Intakes.aspx

(34) Vercellotti J R, St Angelo A J, S̄panier A M. Lipid oxidation in foods: An overview. In: St Angelo AJ, ed. Lipid Oxidation in Food. Washington, D.C.: ACS Symposium Series-American Chemical Society; 1992. p. 1-11

(35) Food and Agriculture Organization of the United Nations. World Health Organization. Codex Alimentarius: Codex Standard for Named Vetable Oils CX-STAN 210-1999; 2017 [cited 12 October 2018]. Available from: https://mvo.nl/media/ voedselveiligheid/codex_standard_named_vegetable_oils.pdf

(36) Van der Merwe GH. Quality parameters for the prediction of mono- and polyunsaturated oil shelf-life. PhD. [Dissertation]. Pretoria: Faculty of Natural and Agricultural Sciences, Department of Food Science, University of Pretoria; 2003.

(37) Troller JA, Christian JHB. Water activity and Food. 1st ed. New York; Academic Press, 1978. Chapter 5: Microbial Growth; $p$ 86-102.

(38) Limbardo RP, Santoso H, Witono JR. The effect of coconut oil and palm oil as substituted oils to cocoa butter on chocolate bar texture and melting point. In American Institute of Physics Conference Series 2017: AIP Publishing; 1840. p. 060001-1 - 060001-12. Available from: https://doi. org/10.1063/1.4982281

(39) Molina-Peralta A, Mach N. Alimentos ricos en ácidos grasos $\omega$-3 libres de contaminantes y aptos para vegetarianos, y su importancia en el desarrollo neurológico normal. Rev Esp Nutr Hum Diet. 2014; 18(2): 89-99.

(40) ljabadeniyi OA, Pillay Y. Microbial Safety of Low Water Activity Foods: Study of Simulated and Durban Household Samples, ]. Food Qual. 2017 [cited 2018 Dec 20]; 2017: Article ID 4931521, 7 pages. Available from: https://www.hindawi.com/journals/ jfq/2017/4931521/cta/

(41) Mozingo RW, O'keefe SF, Sanders TH, Hendrix KW. Improving shelf life of roasted and salted inshell peanuts using high oleic fatty acid chemistry. Peanut Sci. 2004; 31(1): 40-5.

(42) da Silva EP, Siqueira HH, Damiani C, Vilas Boas EV. Physicochemical and sensory characteristics of snack bars added of jerivá flour (Syagrus romanzoffiana). Food Sci. Technol. 2016; 36(3): 421-25. 